\title{
Stratégie et géopolitique de l'opposition à la conservation de la nature : le cas de l'ours des Pyrénées
}

\section{Strategy and geopolitics of the opposition against the preservation of nature: the bear in the Pyrenees}

\author{
Farid Benhammou *,1, Laurent Mermet ${ }^{2}$ \\ École nationale du génie rural, des eaux et des forêts RGTE - Département Environnement, \\ 19, avenue du Maine, 75732 Paris cedex 15, France
}

Reçu le 24 janvier 2003 ; accepté le 4 septembre 2003

\author{
MOTS CLÉS \\ Conservation ; \\ Nature ; \\ Ours (Ursus Arctos) ; \\ Analyse stratégique ; \\ Géopolitique ; \\ Conflits ; \\ Pyrénées
}

\begin{abstract}
Résumé Les oppositions aux actions de conservation de la nature sont souvent présentées, de manière trop schématique, comme les réactions spontanées d'acteurs « locaux » à des initiatives portées par des institutions ou des groupes de pression environnementaux « extérieurs ». Dans cet article, un cas est présenté : les mouvements d'opposition aux actions de conservation de l'ours des Pyrénées, pendant la période 1997-2001. Son analyse montre que l'affichage de cette résistance comme locale relève d'une posture construite par des acteurs qui ne sont pas significativement plus «locaux » que les partisans de l'ours. Les actions de ces opposants relèvent plutôt d'une stratégie socioprofessionnelle et politique pour le maillage et le contrôle du territoire, pour l'appropriation et la maîtrise de postes de pouvoir institutionnel et de ressources financières publiques. Sur cette base, on montre le caractère mystificateur d'analyses qui tendent à attribuer la résistance aux actions de conservation uniquement à des erreurs de la part des acteurs qui les portent. Ces résistances ont leurs dynamiques propres. Elles doivent être analysées de façon précise pour alimenter la réflexion sur les difficultés rencontrées par les actions en faveur de la nature. Les auteurs proposent pour cela une double perspective de gestion stratégique et de géopolitique, complétée par une nécessaire critique du local (ou, du moins, de certaines instrumentalisations de cette notion très flexible).
\end{abstract}

(c) 2003 Éditions scientifiques et médicales Elsevier SAS. Tous droits réservés.

\footnotetext{
${ }^{*}$ Auteur correspondant.

Adresses e-mail : benhammou@engref.fr (F. Benhammou), mermet@engref.fr (L. Mermet).

${ }^{1}$ Géographe

2 Science de la gestion
} 


\section{KEYWORDS}

Conservation project; Brown bear (Ursus Arctos);

Pyrenees mountain; Actors interactions; Geopolitics; Strategy; Conflict

\begin{abstract}
Opposition to conservation initiatives is often presented as a spontaneous reaction of local people to projects or programs promoted by outside environmental institutions or pressure groups. This paper shows that such interpretations, unless based on precise field-work from relevant theoretical perspectives, can be very misleading, often purposefully so. The argument rests on three complementary perspectives : (1) a strategic management approach that sees conservation projects as promoting change, and thus arousing a potential of resistance to change, (2) a geopolitical perspective, focusing on initiatives by given political networks or powerful economic sectors to use this potential resistance to change as a fulcrum for strategies aimed at enhanced control of territories and financial resources from public funds, and (3) a critique of the rhetorics of locality, so often used as a veil for such strategies. A detailed case-study of one emblematic case - opposition to conservation of the brown bear (Ursus arctos) in the French Pyrenees mountains - shows that precisely such strategies are used by an alliance of farming unions and powerful political forces, and play a central role in the evolution of the management of that species. Based on the case study and on the theoretical perspectives they advocate, the authors stress that the many difficulties met by conservation projects and programs should not be ascribed cursorily to bad design or implementation, at least not before an in-depth analysis of initiatives from political, social or economic actors that may have an incentive in bringing about failure of conservation projects and programs.
\end{abstract}

(c) 2003 Éditions scientifiques et médicales Elsevier SAS. Tous droits réservés.

La revue NSS aime à diversifier les points de vue sur un problème. À propos de la préservation d'une espèce en voie de disparition et de sa réintroduction, il n'est question ici ni de biologie des populations, ni de conceptions de la nature qui s'affronteraient, mais de stratégies, de géopolitique, de conflits, d'alliances. Ayant choisi d'étudier les acteurs opposés à l'ours, les auteurs tirent des conclusions qui bousculent les idées courantes, à savoir que, dans l'analyse des conflits environnementaux, les mesures de conservation de la nature sont censées être défendues par des instances supérieures auxquelles s'opposent souvent des intérêts locaux. En insistant sur le caractère quelque peu mystificateur de cette assertion, ils ouvrent ainsi un débat auquel Sophie Bobbé apporte sa contribution. La Rédaction.

Dans l'analyse des conflits environnementaux, une place de premier plan est souvent attribuée à des oppositions entre des acteurs «locaux » et des institutions centralisatrices lointaines : l'État, «l'Europe », des groupes de pression extérieurs. Ce type d'analyse est particulièrement prégnant dans le domaine de la protection de la faune, de la flore et des milieux naturels, que ce soit dans les discours de certains acteurs ou dans les écrits de chercheurs $^{3}$. Il conduit à attribuer systématiquement les conflits et les blocages suscités par les mesures de conservation et de gestion des écosystèmes à des mouvements sociaux porteurs des visions et des intérêts locaux, face à des erreurs ou des abus de la part des acteurs du secteur environnemental. Nous essaierons ici de montrer qu'une telle perspective est insuffisante, voire mystificatrice. En effet, les résistances aux programmes de

\footnotetext{
${ }^{3}$ On trouvera dans un ouvrage de Rossi (2001) un compendium des argumentaires allant dans ce sens.
}

conservation de la nature ont leur dynamique propre (Rowell, 1996). Leur analyse précise est indispensable pour comprendre les difficultés rencontrées par les programmes de conservation.

Nous nous appuierons sur l'analyse d'un cas : la conservation de l'ours brun dans les Pyrénées françaises. Ce dossier avait déjà été abordé lors de précédents travaux (Mermet, 1998, 2001 et 2002). Nous avions ainsi pu suivre les évolutions et rebondissements complexes et incessants de ce dossier tout au long de quatre décennies. À partir de cette base, différents aspects ont été approfondis. Nous avions montré en particulier comment, sur la zone béarnaise, la mise en place d'une structure de gestion (l'IPHB, Institution Patrimoniale du HautBéarn) était présentée comme un dispositif innovant d'intégration et de concertation. En fait, cette institution a principalement permis à un ensemble d'intérêts sectoriels agricoles et d'acteurs politiques locaux d'acquérir des positions très fortes sur le plan du contrôle des financements publics et sur le plan politique, tout cela, sans bénéfice tangible pour la conservation de l'ours. Avec le travail pré- 
senté ici, nous nous sommes tournés vers l'approfondissement d'un autre volet de ce dossier. Nous avons conduit une nouvelle recherche de terrain (Benhammou, 2001) pour analyser les rôles et les dynamiques des porte-parole qui relaient les points de vue favorables, ou au contraire défavorables, à la conservation de l'ours à l'échelle des Pyrénées (territoires des ours autochtones et des ours issus d'une réintroduction en Pyrénées centrales). Les débats étudiés sont ceux qui peuvent influer sur la réception et l'impact des actions de conservation : réunions publiques, associatives, socioprofessionnelles, prises de position dans la presse, consultations organisées par l'État, etc. Quant aux porteparole, les résultats des recherches antérieures nous ont conduits à accorder une attention particulière (mais pas exclusive) aux acteurs d'environnement (services déconcentrés de l'État, associations environnementales...), aux réseaux socioprofessionnels agricoles et aux acteurs politiques. Une cinquantaine d'entretiens semi-directifs approfondis ont été conduits, d'avril à juillet 2001, sur quatre départements pyrénéens : les PyrénéesAtlantiques, les Hautes-Pyrénées, la HauteGaronne et l'Ariège. Le choix des personnes interrogées a été effectué de manière à représenter un éventail de positions - des plus favorables aux plus défavorables - vis-à-vis d'un projet de référence : « le rétablissement d'une population viable d'ours dans les Pyrénées ». L'enquête de terrain a été complétée par l'étude de documents émanant d'administrations comme la Diren Midi-Pyrénées, de structures comme l'Institution Patrimoniale du Haut-Béarn et d'associations favorables ou opposées à l'ours. Nous avons également passé en revue la presse tant locale que nationale. Les résultats de ce travail de terrain portent aussi bien sur les mouvements favorables à l'ours que sur ceux qui y sont opposés. C'est ce deuxième volet que nous souhaitons ici présenter et analyser.

Dans un premier temps, nous préciserons les questions qui nous ont guidés sur ce thème et les perspectives théoriques que nous avons adoptées. Ensuite, nous présenterons une vue d'ensemble de l'évolution du dossier de la conservation de l'ours et de l'émergence des mouvements qui s'y opposent. Nous pourrons alors aborder et discuter successivement les résultats de la recherche qui nous semblent éclairer la question du caractère particulièrement local (ou non) et spontané (ou non) de ces mouvements. En conclusion, à partir de ces résultats concernant le cas de l'ours, nous tirerons des perspectives plus générales sur les questions que soulève l'analyse des oppositions aux actions de conservation de la nature.

\section{Des oppositions locales et spontanées, ou des stratégies politiques et sectorielles délibérées?}

Notre analyse est guidée ici par les deux interrogations suivantes :

- Dans quelle mesure la résistance à des actions de conservation peut-elle être considérée comme locale ? Le fait de mettre en avant des oppositions entre acteurs locaux et nationaux ne masque-t-il pas d'autres oppositions plus déterminantes, en particulier des conflits inter- et intra-sectoriels où se trouve engagé le monde agricole, ainsi que des rivalités politiques pour la maîtrise du territoire et des financements publics?

- Jusqu'où peut-on attribuer à cette résistance un caractère spontané ? A-t-on affaire à des mouvements sociaux en réaction aux discours et actions de protection ? Ou bien l'opposition est-elle plutôt le fait d'organisations porteuses d'autres projets ? Dans ce deuxième cas, la mise en échec des actions de conservation est-elle une condition de réussite de ces projets (par exemple, lorsqu'un projet technique donné n'est pas compatible avec la présence durable d'une espèce animale) ? Ou bien devrait-elle être analysée comme un levier d'action politique ou syndicale?

Sur le plan théorique, ces questions nous paraissent devoir être abordées dans une triple perspective.

Elles relèvent d'abord d'une analyse de la gestion de l'environnement en termes stratégiques. Comme nous pensons l'avoir déjà montré (Mermet, 1992 et 1998), ce n'est pas seulement pour des raisons conjoncturelles (erreurs dans l'action, contextes « difficiles »... ) que l'action en faveur de tel ou tel objet environnemental suscite des résistances, mais pour des raisons structurelles. En effet, ces actions doivent être considérées comme des projets portés par un acteur (ou un secteur des pouvoirs publics) pour conduire d'autres acteurs (ou secteurs) à changer leur pratique ou leurs projets. Dès lors, les dynamiques de résistance au changement sont inhérentes, au moins dans leur principe, à ces actions. Les acteurs porteurs du projet environnemental se trouvent pris dans des jeux antagonistes (au moins latents, ou sousjacents) dans le cadre desquels ils doivent déployer des stratégies pour prévenir les oppositions délibérées, ou y faire face si elles surviennent malgré tout. C'est ce qui nous fait qualifier de « stratégique " cette perspective théorique. Elle contraste avec d'autres approches, souvent prô- 
nées aujourd'hui, qui semblent partir plutôt de l'hypothèse que la conservation n'est légitime que fondée sur une action conjointe de tous les acteurs concernés. Dans cette optique, les oppositions traduiraient au mieux des erreurs commises par les acteurs qui portent les projets de conservation. Au pire, elles seraient le signe d'un abus de pouvoir de leur part, puisqu'ils auraient essayé de surmonter des résistances qu'il faudrait a priori considérer comme légitimes. Dans tous les cas, ils n'auraient nullement à déployer des stratégies à l'égard des autres acteurs. Les résultats de la recherche présentée ici contribuent selon nous à montrer les limites de telles approches.

En synergie avec cette perspective de gestion stratégique, la question des oppositions à la conservation de la nature relève ensuite d'une approche géopolitique. En effet, celle-ci permet de mettre à leur juste place la dimension conflictuelle et la dimension territoriale inhérentes à la gestion de la biodiversité, aux usages des ressources, aux aménagements des milieux naturels. Une situation géopolitique se définit, selon Y. Lacoste (1995), par des rivalités de pouvoirs, par des rapports entre des forces mettant en jeu des territoires. Traditionnellement, la géopolitique traite d'enjeux à l'échelle internationale, notamment en ce qui concerne les rivalités entre États (géopolitique externe). Depuis quelques années, on parle aussi de géopolitique concernant des problèmes politiques au sein d'un même État, par exemple sur des thèmes comme les revendications régionalistes ou les questions d'aménagement du territoire (Lacoste, 1995, 1997). Cette approche de géopolitique interne nous paraît pertinente pour étudier comment des groupes, des organismes, se structurent géographiquement, organisent leur force, s'opposent à propos de questions de conservation de la nature au sein du territoire national. La dimension « territoire » (géo) est importante, mais la dimension « pouvoir » (politique) l'est peut-être encore davantage : politique dans le sens de la conduite des débats et des affaires publiques, mais aussi dans le sens de la lutte pour la conquête ou la conservation des positions de pouvoir dans les institutions politiques.

Enfin, ces analyses en terme de gestion stratégique et de géopolitique doivent être complétées c'est un préalable ou un travail nécessaire d'accompagnement - par une interrogation critique sur le local. En effet, pour simpliste qu'elle soit, la vision qui oppose des paysans ou des « montagnards » à un État oppresseur au service « d'écolos urbains » est aujourd'hui très prégnante, que ce soit dans le champ de l'action (le travail de terrain présenté ici l'a encore montré), ou dans le champ académique. Dans le cadre d'une certaine idéalisa- tion, cette vision réduit d'ailleurs le local à sa définition d'opposition à l'État, lui-même réduit à des fonctions primaires (le maintien de l'ordre, l'apport de financements...), l'échelle de la « démocratie vraie » étant dorénavant vue exclusivement dans ce «local » idéalisé (Bourdin, 2000). Cela participe de ce que cet auteur nomme la "vulgate localiste ${ }^{4}$. Pour sortir de telles visions caricaturales, il convient d'adopter une perspective qui considère le local comme traversé par une diversité de champs et d'acteurs (économiques, politiques, administratifs, associatifs, etc.) (Djouldem, 1991).

Sortir ainsi d'une conception caricaturale du local contribue, on le verra, à rendre à ces conflits environnementaux leurs véritables dimensions stratégiques et géopolitiques.

\section{Stratégies et contre-stratégies autour de la conservation de l'ours des Pyrénées}

\section{À l'origine, des ours, des hommes, deux théâtres : le Béarn et les Pyrénées centrales}

Au fil des années, le dossier de l'ours des Pyrénées a fini par devenir emblématique des problèmes de conservation de la faune en France. Déjà ancien, le déclin de la population d'ours s'est particulièrement accéléré au XXe siècle (voir graphique) (Camarra et Parde, 1992 ; Caussimont, 1993) et cette espèce, dont l'aire de répartition s'est réduite en ilôts éloignés et de plus en plus petits, se trouve aujourd'hui dans une position critique dans notre pays. La population actuelle d'ours, d'une quinzaine d'individus, provient de deux origines différentes, si bien que jusqu'à une époque très récente, le dossier de l'ours recouvrait deux volets tout à fait distincts.

Le volet béarnais correspond à un reliquat de population autochtone, qui regroupait moins d'une

\footnotetext{
${ }^{4}$ Cette synthèse d'idées reçues se compose le plus souvent de trois ingrédients qui permettent l'affirmation radicale de la primauté du local et la valorisation systématique des petites entités communautaires territoriales :

- un héritage partagé (toute spécificité du groupe est exagérée et utilisée comme un instrument de résistance) ;

- la « modernité », jugée dangereuse (les mesures de protection de l'environnement sont présentées comme une ingérence de la société moderne et non les aménagements, l'exploitation non durable des ressources souvent activement promus par des lobbies pourtant non locaux) ;

- la foi dans la proximité (Small is beautiful) : ceux qui parlent au nom de l'échelle locale sont forcément dans le vrai, l'authentique, la légitimité (Bourdin, 2000).
} 
dizaine d'individus au début des années 90 , exclusivement concentrés en Béarn. Aujourd'hui, il ne comporte vraisemblablement plus que cinq ours, dont une seule femelle (Diren 2002). Sans intervention, la population est condamnée à l'extinction. Les tentatives des années 80 pour mettre en place une gestion concertée de cette population béarnaise d'ours («Plan Ours » de 1984, « Directive Ours » de 1988) se sont heurtées à une résistance obstinée, s'appuyant sur le ministère de l', ses services et établissements publics. Les conflits intenses qui en ont découlé ont défrayé la chronique dans les années 80 et jusqu'en 1991, culminant avec la « guerre de l'ours » qui résultait, entre autres, de la mise en réserve d'un espace minimum vital pour la préservation des derniers ours pyrénéens, les « réserves Lalonde » (Dendaletche, 1993). Le climat est alors d'autant plus agité qu'en 1990-1992, les antagonismes au sujet de l'axe routier E7 et du tunnel du Somport en vallée d'Aspe viennent se surajouter. Ce dossier environnemental conflictuel, qui a souvent été stratégiquement mêlé à celui de l'ours, procède en effet du même contexte géopolitique. En effet, des acteurs politiques locaux béarnais reprennent à leur compte des aménagements promus au niveau national et européen, et ce, contre l'intérêt public local (Soubeyran et Barnier, 1996). À la suite de ces conflits, certains acteurs locaux (élus, chasseurs, socioprofessionnels agricoles), activement aidés par des services du ministère de l'Agriculture, remportent l'épreuve de force en 1993 et obtiennent que leur soit confiée la gestion de l'ours et des aménagements qui nuisent aux espaces naturels nécessaires à la conservation de l'espèce. À cette fin, un syndicat mixte, l'Institution Patrimoniale du HautBéarn (IPHB) est créé en 1994 (Mermet, 2001 et 2002). Les acteurs locaux et nationaux favorables à l'ours sont alors largement écartés du dossier de l'ours en Béarn. Ils voient s'éloigner la perspective d'un renforcement de population jugé pourtant nécessaire par tous les experts (ASCA et Servheen, 1996).

Un deuxième volet du dossier ours s'ouvre alors dans les Pyrénées centrales. En effet, un groupe d'élus locaux de la Haute-Garonne montagnarde, encouragé par Artus, une importante association de défense de l'ours, décide de créer en 1991 l'ADET (Association pour le développement économique et touristique de la haute vallée de la Garonne, puis par la suite des Pyrénées centrales). Le but de l'association est de réintroduire des ours afin de valoriser économiquement et écologiquement cette zone rurale fragile. Cependant, jusqu'en 1993, le ministère de l'Environnement est réticent à l'égard de ce projet. Selon un principe de biologie de la conservation, il est préférable (quand c'est possible) de renforcer les noyaux de population relictuels existants, plutôt que de réintroduire là où l'espèce concernée a disparu (à l'époque, il ne resterait qu'un seul ours dans les Pyrénées centrales). À partir de 1993, le blocage de la situation dans le Haut-Béarn conduit le ministère à changer de stratégie. Celui-ci souhaite, selon les termes d'un de ses responsables, ouvrir un " deuxième front » et soutient alors le dossier de l'ADET, qui, dès lors, prend forme assez rapidement. Une réintroduction de trois ours de Slovénie, deux femelles et un mâle, a donc lieu en 1996 et 1997. Les lâchers sont faits dans la forêt de Melles en Haute-Garonne (Fig. 1). Hasard, les femelles étant pleines, quatre oursons issus des deux portées survivent, une des mères est abattue par un chasseur, la femelle restante a eu deux oursons en 2000 , deux autres en 2002 et le grand mâle slovène s'ajoute à un effectif qui est de l'ordre de 10 individus (Diren, 2002). Ces ours, notamment les jeunes, sont beaucoup plus mobiles que certains ne l'avaient prévu, si bien qu'ils se trouvent répartis des Pyrénées-Atlantiques aux Pyrénées-Orientales, soit sur toute la chaîne des Pyrénées. C'est ce deuxième volet du dossier qui défraye régulièrement la chronique depuis 1997, donnant lieu à la nouvelle vague de mobilisation anti-ours sur laquelle nous concentrerons ici l'analyse.

\section{Géographie des mobilisations locales anti-ours : complexification des deux tableaux (cf. encadré 1)}

La première mobilisation locale hostile à la réintroduction d'ours est celle qui s'est déclarée dans l'immédiate proximité géographique de l'ADET. Il s'agit de l'ADIP, Association de défense de l'identité pyrénéenne. Elle avait été encouragée par les chasseurs du milieu des années 80 au début des années 90 , avant que ceux-ci ne se rallient progressivement, de 1993 à 1995, au projet de réintroduction de l'ADET et d'Artus. En 1999, l'association anti-ours ADIP est réactivée par un groupe d'éleveurs et d'élus de la région de Luchon (HauteGaronne), car les ours ont commencé à se déplacer en Luchonnais, en Barousse (Hautes-Pyrénées) et en Castillonnais (Ariège) (Fig. 1).

À partir de 1999, un groupe d'éleveurs ariégeois soutenu par des élus, en particulier le député Augustin Bonrepaux, cherche à promouvoir l'opposition à l'ours à travers les structures syndicales départementales (FDSEA, Confédération paysanne, Fédération Pastorale au sein du Conseil général de 


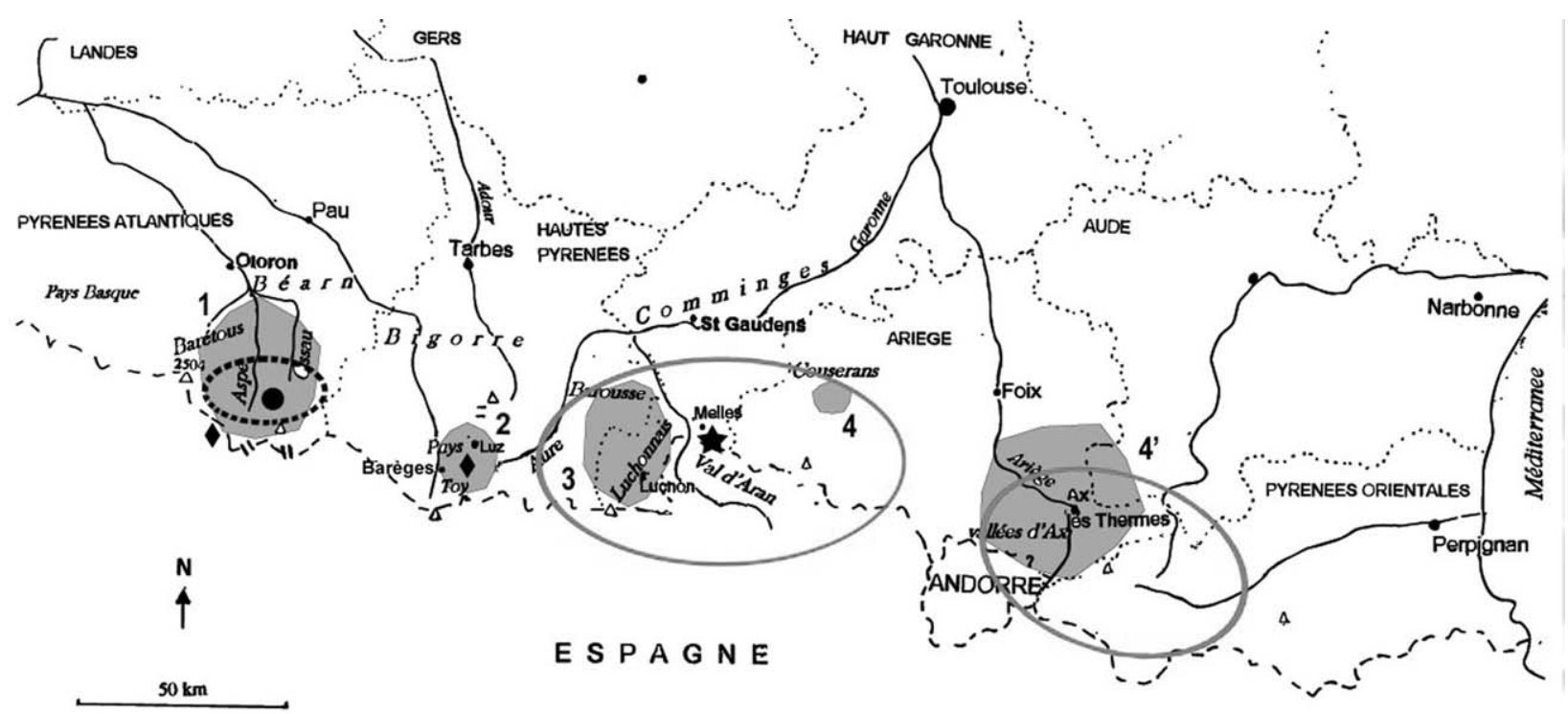

Aires de répartition des ours bruns et des principales composantes de I'ADDIP-IDAMP (2001)

\author{
Zones grises : \\ 1. Association des Eleveurs Transhumants des trois vallées \\ et comité contre la réintroduction d'ours étrangers \\ 2. ASPP \\ 3. ADIP \\ 4. Groupe d'éleveurs et d'élus ariègeois \\ 4'. Fief électoral de A. Bonrepaux
}

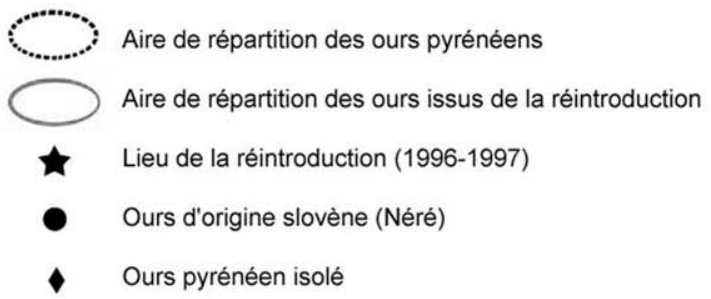

Réalisation F.Benhammou, 2003

Sources : Réseau Ours Brun / DIREN-MP / Benhammou (2001)

Figure 1 Aires de répartition des ours bruns et des principales composantes de l'ADDIP-IDAMP (2001)

l'Ariège ${ }^{5} \ldots$... Ils organisent alors plusieurs actions, dont une manifestation significative à Foix en août 1999. En 2000, avec les déplacements des jeunes ours, quelques représentants agropastoraux professionnels de l'Aude et des Pyrénées-Orientales les rejoignent sans s'investir véritablement.

À partir de 2000, avec la progression d'un ours subadulte d'origine slovène, Néré, vers l'ouest et le cœur des Hautes-Pyrénées, une forte opposition se structure notamment dans le Pays Toy (région de Barèges, Luz-St-Sauveur, voir carte) autour d'élus comme le maire de Luz-St-Sauveur, certains chas-

\footnotetext{
${ }^{5}$ Par le biais de la Fédération pastorale de l'Ariège, le Conseil général a absorbé les missions pastorales de la DDAF à la fin des années 1980 dans le cadre de la décentralisation. Ce service a été structuré par un ancien contractuel de la DDAF. Grâce à cet organisme, l'Ariège, bien que victime de la crise de l'élevage, a été pionnière en terme de gestion des espaces pastoraux de montagne. Elle a travaillé notamment à l'organisation des éleveurs en associations foncières et groupements pastoraux et quadrille ainsi facilement le territoire pastoral. Cette Fédération représente un outil de contrôle politique majeur du tissu social agricole et concrétise, pour l'Ariège, l'alliance entre une partie de la profession agricole et le groupe dominant de la vie politique ariégeoise.
}

seurs et la responsable du syndicat ovin local Barèges-Gavarnie. Pour regrouper les forces hostiles à l'ours, ils créent l'ASPP, Association pour la sauvegarde du patrimoine pyrénéen. En poursuivant sa route vers l'ouest, Néré arrive en Béarn en 2000, d'abord aux marges, puis bientôt au centre de la zone de compétence de l'Institution patrimoniale du Haut-Béarn.

Dès lors, les deux volets du dossier se trouvent réunis dans une configuration nouvelle, favorisant une dynamique couvrant l'ensemble du massif pyrénéen. Cette première étape de contestation culmine en mars 2000, lorsque le député A. Bonrepaux fait voter à l'Assemblée nationale un amendement à une loi sur la chasse. Cet « amendement Bonrepaux » prévoit l'arrêt de l'opération de réintroduction et le retrait des grands prédateurs des territoires de montagne. Bien qu'il ait été vidé de son contenu majeur par le Conseil constitutionnel, cet amendement, par son impact médiatique considérable, a eu le temps de déstabiliser les administrations de l'Environnement à toutes les échelles. Il dynamise l'opposition à l'ours et lui donne un nouvel élan structurant à l'échelle pyrénéenne. 


\section{Encadré 1}

Le paysage associatif anti-ours ${ }^{6}$

\section{Associations locales (à l'échelle de quelques vallées, quelques cantons)}

\section{- En Béarn (Pyrénées-Atlantiques)}

- Association des éleveurs transhumants des trois vallées : Ossau, Aspe et Barétous (AET3V) :

Créée en 1995 pour représenter les bergers au sein de l'IPHB. Dès l'origine hostiles à l'ours, ses dirigeants voyaient avant tout celui-ci comme un prédateur peu compatible avec l'extensification du système pastoral béarnais. De plus, les mesures de conservation de l'espèce contrarient l'équipement de la montagne en piste sylvo-pastorale. Deux porte-parole étaient particulièrement virulents et s'opposaient à une réintroduction en Béarn. Écartés de l'association en 2002 en raison de leur radicalisme, ils s'investissent maintenant exclusivement dans l'association pyrénéenne hostile à toute réintroduction d'ours, l'IDAMP.

- Comité de défense contre la réintroduction d'Ours étranger :

Créée en 1996 contre tout renforcement de population d'ours. Des élus de la vallée d'Ossau, historiquement hostiles à l'ours (J. Baylocq, maire de Bielle), en sont à l'origine. Avec l'arrivée d'un ours d'origine slovène, ils n'hésitent pas à diffuser une rhétorique xénophobe (Benhammou, 2003).

- En Haute-Garonne

- Association pour développement de l'identité pyrénéenne, ADIP :

Créée au début des années 1990 par des chasseurs et quelques éleveurs pour fédérer les énergies contre les mesures de protection des ours (Réserves Lalonde) et le projet naissant de réintroduction en Pyrénées centrales. Redynamisée en 1999-2000 par des élus du Luchonnais, de l'est des Hautes-Pyrénées et quelques éleveurs.

- En Hautes-Pyrénées :

- Association pour la sauvegarde du patrimoine pyrénéen, ASPP :

Créée en 2000 suite au passage d'un ours issu de la réintroduction. L'ASPP recouvre le territoire du Pays Toy (région de Barèges et de Luz-St-Sauveur) dans les Hautes-Pyrénées. Les acteurs de cette association s'avèrent les mêmes que ceux étant à l'origine du syndicat ovin d'une AOC viande de mouton en cours de promotion (Barèges-Gavarnie).

Associations fédérales et régionales :

- Association pour le développement durable de l'identité pyrénéenne, ADDIP :

Créée en 2000 sous l'impulsion d'un groupe d'élus et d'éleveurs de la Confédération paysanne proches du député Augustin Bonrepaux. Ils ont pris pour modèle et absorbé l'ADIP de HauteGaronne. L 'ADDIP cherche à réaliser la fédération de tous les organismes, structures, conseils municipaux, associations opposées à la présence des ours et à la réintroduction à l'échelle des Pyrénées.

- Association interdépartementale de défense de l'agriculture des montagnes pyrénéennes, IDAMP : Créée en 2000, parallèlement à l'ADDIP dont les objectifs sont proches. Elle tente de faire une fédération d'organismes à vocation agricole uniquement (chambres d'agriculture, syndicats agricoles, groupements pastoraux...). Cette association est opposée à toute forme de cohabitation avec des prédateurs sauvages.

\footnotetext{
${ }^{6}$ Il peut paraître simplificateur de parler d'anti-ours comme de pro-ours. Outre un souci de concision, il nous est néanmoins clairement apparu, au regard des statuts et des objectifs des associations en question, que l'hostilité ouverte, l'opposition à l'ours et à sa réintroduction étaient des éléments majeurs de leur mobilisation. Plusieurs militants eux-mêmes se qualifient d'anti-ours (Benhammou, 2001). Dans ce texte, il est question d'opposition à l'ours et d'opposition à la réintroduction de l'ours. Nous avons clairement identifié dans notre travail que ces oppositions étaient les deux manifestations d'un même phénomène (Ibid). Les discours des uns se prononçant contre une réintroduction, mais pas contre l'ours pyrénéen, relevaient d'une stratégie rhétorique ne résistant pas à l'analyse des actes et des alliances (Ibid; Benhammou, 2003).
}

Retracée ici dans ses grandes lignes et sur l'ensemble de la chaîne, l'évolution récente du dossier complète l'historique dont nous disposions déjà jusqu'en 1996 sur la seule zone béarnaise (Dendaletche, 1993 ; Mermet, 1998). L'initiative des acteurs d'environnement en Pyrénées centrales a conduit, entre 1997 et 2001, à une reconfiguration radicale du dossier, avec l'expansion géographique spectaculaire de la zone concernée et l'implication d'un grand nombre d'acteurs nouveaux. Dans cette nouvelle étape de la construction d'un système de gestion de l'ours, nous constatons une fois de plus 
le rôle déterminant que joue l'adaptation stratégique des acteurs du secteur environnemental aux oppositions actives qu'ils rencontrent. S'ils n'avaient pas ouvert délibérément, dans les années 90 , un « deuxième front » dans les Pyrénées centrales, la situation serait aujourd'hui caractérisée par un nombre d'ours nettement inférieur, réparti sur une zone géographique bien plus restreinte. Si cette nouvelle situation paraît plus favorable à la conservation de l'ours, elle se trouvait aussi face à des formes nouvelles d'opposition. Nous allons en proposer une analyse stratégique et géopolitique plus approfondie.

Au préalable toutefois, comme nous l'avons exposé plus haut, il nous faut lever l'hypothèque d'une analyse simpliste de ces mouvements comme réaction « locale ", en réponse à des acteurs environnementaux qui, eux, ne le seraient pas.

\section{Composition du mouvement anti-ours récent : est-il particulièrement « local »?}

Dans le cadre des conflits géopolitiques, les forces en présence jouent des représentations selon une double acception du terme : d'une part, elles cherchent à véhiculer une certaine vision du monde ; d'autre part, certains acteurs n'hésitent pas à se mettre en scène (Lacoste, 1995). Ainsi, les opposants à l'ours se posent-ils souvent en victimes. Victimes d'une réintroduction qu'ils n'ont pas demandée et/ou d'une politique de conservation spatialement trop contraignante. Ils affirment parler au nom des « locaux » - ici, des «Pyrénéens » - qui sont censés se sentir représentés par leur discours (cf. Diren, 2000 : propos de Bonrepaux et Bedos). Comme l'exprime une éleveuse responsable d'une association anti-ours : "On sent bien que ce sont nos territoires qui sont convoités pour servir à autre chose [...]. On a trop laissé la parole à d'autres qui n'avaient pas nos références historiques, culturelles, à d'autres qui n'étaient pas issus du milieu agricole, rural pyrénéen, il fallait qu'on reprenne l'initiative [...]. C'est Nous les Pyrénées, c'est pas eux qui vont nous imposer ça ». En regard de ce discours, nous avons examiné sur la base de notre matériau d'entretien l'origine et la trajectoire des principaux leaders anti-ours qui ont occupé le premier plan en 2001-2002. Il ressort qu'ils ont en commun un profil de néo-ruraux, ils ont fréquemment fait des études supérieures, ils ont un passé de militant expérimenté et adoptent des discours anti-ours souvent très virulents. Retenons simplement ici quatre profils de responsables d'associations opposées à l'ours ${ }^{7}$.

- Monsieur W a été élu à la tête de son association en tant qu'éleveur. Il n'est pas issu du milieu rural agricole et possède une formation de dessinateur en bâtiment et génie civil. Pour lui, « l'élevage est une passion ». En effet, ses revenus principaux proviennent de son activité hivernale de moniteur de ski. Il a été encouragé à s'engager davantage dans le combat anti-ours, surtout depuis la dynamique insufflée par A. Bonrepaux.

- Madame X est aussi une proche (politiquement) d'A. Bonrepaux. C'est une éleveuse de vaches et d'ânes, originaire de l'Ouest et de la région parisienne, où elle a acquis une formation d'ingénieur agricole. Elle s'est installée en Ariège avec son conjoint comme beaucoup de néoruraux à la fin des années 70 . Membre de la Confédération paysanne de l'Ariège, elle vit surtout de la location d'ânes pour les randonneurs ou le transport par bât.

- Monsieur $\mathrm{Y}$, un responsable syndical agricole départemental particulièrement remarqué pour ses positions radicalement anti-ours, s'est installé dans le département au début des années 80 , venant d'une autre région, et d'un autre milieu professionnel.

- Madame Z présente un itinéraire particulièrement éclairant. Originaire du Massif central, elle a mené des études supérieures en sociologie rurale. Elle a entrepris des recherches et une thèse sur un terroir des Pyrénées. C'est là qu'elle a épousé un éleveur. Après avoir travaillé dans le secteur social, elle est devenue chef d'exploitation à plein temps et s'est impliquée activement dans les structures socioprofessionnelles du département. Quand un ours issu de la réintroduction est passé dans sa petite région, elle s'est systématiquement mise en avant dans le discours médiatique contre l'ours dans son département.

Comme le confie un berger des Hautes-Pyrénées, ces responsables (parmi lesquels l'auteur des propos cités plus haut sur « nos territoires ») sont « plus pyrénéens que les Pyrénéens eux-mêmes ». Cette remarque rejoint les analyses d'Yves Lacoste (1997) sur certains mouvements régionalistes en France qui sont parfois activement portés par des gens n'ayant pas leurs « racines » dans la région et n'y étant souvent même pas nés.

\footnotetext{
${ }^{7}$ Nous avons décidé de rendre anonymes les personnes interrogées contrairement aux élus, dont nous citons les noms dans la mesure où il s'agit de personnes publiques s'assumant comme telles.
} 
Sur la base de notre matériau d'enquête, on ne trouve pas de différenciation particulière quant à l'origine géographique, sociale, et quant à la trajectoire personnelle plus ou moins « locale » des opposants et partisans actifs de l'ours (Benhammou, 2001). Les opposants à la conservation de la nature sont juste plus insistants dans la mise en avant d'un certain « localisme ». Au total, plutôt que de réaction locale aux actions de conservation, il nous semble approprié de parler d'une posture identitaire et localiste construite, alimentée et consolidée à l'occasion de l'arrivée d'un animal emblématique, et utilisée en appui à des stratégies militantes, syndicales et politiques. Beaucoup plus éclairants sont les positionnements stratégiques dans les jeux politiques des collectivités territoriales et des organismes syndicaux et socioprofessionnels.

\section{Mouvement social anti-ours ou bien stratégies d'action politiques et syndicales à l'échelle de la chaîne pyrénéenne?}

\section{Un effort politique et syndical d'organisation de l'opposition à l'ours...}

Jusqu'en 2000, on a vu plus haut que le dossier de l'ours couvrait deux théâtres distincts (Béarn - Pyrénées centrales), eux-mêmes abritant nombre de mini-scènes locales à travers les Pyrénées, émiettement que traduit la diversité des initiatives. Outre le rôle joué par l'intrusion de l'ours Néré dans le fief de l'IPHB, les diverses dynamiques syndicales et politiques débouchent sur une organisation de l'opposition à l'ours construite en commun à l'échelle du massif sous deux impulsions politiques et organisationnelles, l'une ariégeoise, l'autre béarnaise.

L'impulsion ariégeoise s'inscrit dans la suite de la première réaction à la réintroduction, déjà relatée, débouchant sur l'activisme du député Bonrepaux et de ses proches, membres des milieux politiques et socioprofessionnels agricoles de l'Ariège. Pour tirer les fruits de l'affaiblissement des positions du ministère de l'Environnement et de la Diren, dû au retentissement national de son amendement, le député ariégeois a souhaité passer de sa stratégie d'opposition frontale à la création d'une structure de gestion pilotée par les collectivités territoriales : un outil de maîtrise politique pouvant drainer des financements publics importants - un projet sans doute inspiré du succès de l'IPHB. Cette prise de position stratégique apparaît notamment dans le discours qu'il prononce à Toulouse en juin 2000 dans le cadre d'un débat public sur l'ours (Diren, 2000). Ce discours passe progressivement d'une attaque directe prolongée et virulente contre l'opération de réintroduction de l'ours à une proposition de gestion négociée. On peut y relever les jalons suivants :

- (Au début) «... les scientifiques nous assurent que les ours tolèrent les activités humaines. C'est heureux, mais cela ne nous rassure pas, et je demande tout de suite que l'on fasse une autre étude pour savoir si les hommes peuvent tolérer les ours. »

- (Au milieu) « ... dans les conditions actuelles de l'élevage, la cohabitation n'est pas possible, ce que je me garderai bien de dire, c'est si elle sera un jour possible. »

- (En conclusion) « ... je termine par une proposition concrète : [...] mettre en place une association pyrénéenne [...] afin de définir ensemble, entre Pyrénéens, quelle peut être l'utilisation la plus efficace des moyens pour lesquels le ministère [chargé de l'Environnement] s'est engagé. [...] À partir de là, élaborer un programme d'actions concrètes pour la valorisation du pastoralisme et de l'identité montagnarde. [...]. Ce n'est qu'à la suite d'une telle démarche et lorsque tout sera organisé comme nous le souhaitons qu'il sera possible alors de dire si la cohabitation est possible. Merci. »

Pour faire réussir cette stratégie, il faut cependant stabiliser l'organisation de l'opposition à l'ours, lui faire prendre une ampleur pyrénéenne et l'inscrire dans la durée, en particulier en la dotant d'une base associative. C'est pour cette raison que l'ADDIP ${ }^{8}$ a été créée en reprenant l'association en latence, l'ADIP.

Cette impulsion ariégeoise en croise une seconde d'origine béarnaise. Elle résulte directement de l'imbrication des deux problématiques « ours béarnais »/« ours issus de la réintroduction ». En s'appuyant sur la dynamique des autres départements contre la réintroduction, des militants syndicaux et associatifs agricoles béarnais, avec le soutien actif d'élus de la vallée d'Ossau, relancent au sein de I'IPHB - qui affiche jusque-là une position ambiguë sur cette question - leur revendication d'une opposition radicale à tout renforcement de population d'ours en Béarn. Pour appuyer cette stratégie, ils décident de se réunir avec des éleveurs des HautesPyrénées et de l'Ariège. En juin 2000, l'Association

\footnotetext{
${ }^{8}$ Association pour le développement durable de l'identité pyrénéenne.
} 
interdépartementale de défense de l'agriculture de montagne pyrénéenne est ainsi créée. L'IDAMP est exclusivement destinée à mobiliser les organisations agricoles, syndicats, chambres d'agriculture. L'ADDIP, elle, est censée être une structure plus ouverte aux autres acteurs locaux (élus, chasseurs, guides...), et non strictement agricoles. La création de l'IDAMP dynamise notamment le foyer récent d'opposition organisé par des éleveurs de Barèges réunis au sein de l'Association de sauvegarde du patrimoine pyrénéen, ASPP. La leader de ce mouvement, localisé au départ, va à son tour représenter un relais efficace de l'IDAMP. Elle est alors promue à la tête de cette association, et fait du combat contre l'ours une véritable croisade dont le but est de faire aboutir un projet socioprofessionnel qui a toujours échoué jusque-là : un organisme agricole fédérateur à l'échelle des Pyrénées.

\section{... qui se trouve entraîné dans une dynamique de radicalisation}

Ces deux impulsions parallèles, ariégeoises et béarnaises, ont tout pour se rencontrer et entrer en synergie, les projets politiques et socioprofessionnels qu'elles portent étant déjà liés par des alliances fortes. Au départ, l'ADDIP et l'IDAMP diffèrent toutefois par leur positionnement stratégique et le discours qui les accompagne : du côté des réseaux politiques ariégeois, une logique gestionnaire de type patrimonial, portée par la Fédération Pastorale de l'Ariège et s'inspirant du modèle IPHB ; du côté de l'IDAMP, une stratégie portée par un discours d'opposition radicale. Le rapprochement en vue d'une organisation à l'échelle du massif s'opère, mais sous les auspices d'une dynamique de radicalisation. Les membres de l'IDAMP, notamment certains éleveurs béarnais ${ }^{9}$ siégeant à l'IPHB, opposent un refus catégorique de la négociation et mettent en jeu le soutien de l'IDAMP à l'ADDIP. Ils ne veulent pas, disent-ils, gérer « l'argent de l'ours » et s'opposent à toute réintroduction, car ils craignent que cela conduise à une augmentation de la population ursine. Ils s'affirment dépassés par la gestion de l'ours à l'IPHB et ne veulent pas promouvoir cette logique, qui suppose notamment de privilégier des systèmes d'élevage compatibles avec la présence des prédateurs. Ces divergences de vue entre les différentes composantes de l'opposition à l'ours créent un flottement qui perdure jusqu'à la fin de l'hiver 2000. Lorsque la Fédération Pastorale de l'Ariège se rend compte que les administrations

\footnotetext{
${ }^{9}$ Ces éleveurs étaient membres de l'Association des éleveurs transhumants des trois vallées: Ossau, Aspe, Barétous.
}

de l'Environnement ne sont pas prêtes à donner des financements importants pour une «IPHB bis », la situation se clarifie. La stratégie gestionnaire et patrimoniale lui paraît alors, semble-t-il, moins attractive.

Finalement au printemps 2001, la position radicale prônée par l'IDAMP l'emporte. Cette association se jumelle à l'ADDIP qui opte elle aussi pour la stratégie d'opposition frontale. Le message sera dur et clair. Les deux associations diffusent en mars 2001 la Colère des Pyrénées, un pamphlet contre la réintroduction de l'ours et les grands prédateurs où des témoignages, principalement d'éleveurs, de socioprofessionnels et de militants agricoles, résument leurs arguments (ADDIP-IDAMP, 2001). Au final, une grande structure à deux têtes s'est créée à l'échelle des Pyrénées, l'ADDIP-IDAMP. Elle a fédéré plusieurs mouvements localisés dans plusieurs « fiefs » (vallées, cantons, pays) qui avaient au départ des stratégies de mobilisation différenciées par rapport à l'ours (plutôt belliqueuses ou plutôt ouvertes à la négociation). Une fois que l'ADDIP et l'IDAMP ont consacré leur partenariat, l'homogénéisation des positions des porte-parole s'est faite sur la ligne dure.

Il est à noter que ce mouvement, qui a réussi à afficher une unité de discours, est porté à l'échelle de l'ensemble des Pyrénées par un petit groupe de personnes bien identifiées, essentiellement des militants syndicaux agricoles très expérimentés, soutenus par certains élus et qui animent ensemble les deux associations ADDIP et IDAMP. La stratégie d'action est mise en œuvre à cette échelle du massif. Pour matérialiser une présence de terrain, le printemps 2001 a vu l'organisation de manifestations médiatisées localisées à des points nodaux ${ }^{10}$ répartis sur toutes les Pyrénées. 150 à 200 manifestants s'y retrouvaient à chaque fois, dont un important noyau dur de militants toujours identiques. L'essentiel était de montrer que le terrain régional était occupé et quadrillé, afin de promouvoir l'image d'un territoire où dominent les opposants à l'ours (Fig. 1).

Ainsi, le dossier de l'ours se trouve aujourd'hui en suspens. D'un côté, les acteurs favorables à sa conservation ont obtenu une évolution significative de la situation de gestion de cette espèce. Ils éprouvent néanmoins (pour des raisons qui dépassent le cadre de cet article) des difficultés à mettre en œuvre une nouvelle stratégie clairement définie. De l'autre, l'opposition à la conservation de

\footnotetext{
${ }^{10}$ À Asson en Béarn, dans la zone temporairement fréquentée par un nouvel ours d'origine slovène, Néré ; à Barèges, la zone de l'ASPP, et à Orlu, le bastion politique des proches d'A. Bonrepaux.
} 


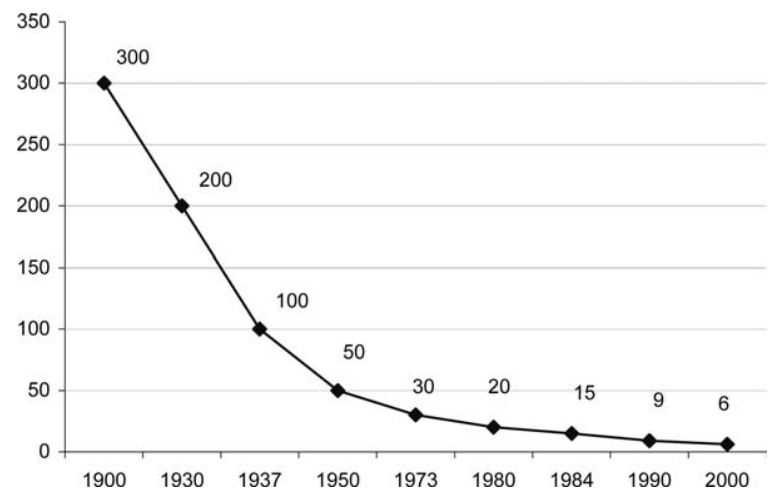

Figure 2 Le déclin de la population d'ours pyrénéens au cours du $X X^{e}$ siècle ( $F$. Benhammou (2003), d'après les données de Camarra et Parde (1992) et De Marliave (2000). Ce graphique ne prend pas en compte les ours issus de la réintroduction)

l'ours est portée par des acteurs politiques et syndicaux bien identifiés, elle est solidement organisée et unifiée à l'échelle de la chaîne pyrénéenne. Cette situation peut être vue comme une forme d'impasse : en effet, le discours d'opposition radicale à la présence de l'ours débouchera difficilement sur un programme d'action réaliste. Mais la situation peut aussi bien être vue comme ouverte, puisque l'on a déjà assisté plusieurs fois, au cours des trois dernières décennies, aux reconversions stratégiques. Celles-ci peuvent conduire de positions d'opposition énergiquement construites à des positions de gestion et de contrôle politique habilement maîtrisées, comme le montre l'exemple de l'IPHB (Fig. 2).

\section{Discussion et perspectives}

Au regard des questions que nous avons soulevées, ces dynamiques organisationnelles à l'échelle du massif pyrénéen appellent trois remarques.

D'abord, la dynamique de radicalisation correspond bien aux logiques stratégiques décrites par Couteau-Bégarie (2000). Cet auteur montre comment, dans un conflit, les diverses mouvances au sein d'un camp peuvent être conduites à se rallier à la position la plus radicale, poussées par le souci de se sentir plus fortes malgré tout et de ne pas passer pour traîtres. Ainsi, le président de l'ADDIP a-t-il renoncé du jour au lendemain au processus de dialogue qui s'était engagé avec l'association environnementale de Haute-Garonne, Nature Comminges. De même, les socioprofessionnels proches de A. Bonrepaux, divisés sur le mode de mobilisation, ont abandonné leur projet patrimonial pour le moment.

Ensuite, l'évolution de l'organisation du mouvement anti-ours correspond de façon frappante à l'analyse proposée par Djouldem de l'organisation locale en réseau. Dans le cas qui nous concerne, en s'opposant à tout ce que l'on associe à l'ours, en adossant cette opposition à un argumentaire identitaire et territorial, un réseau restreint de personnes entreprend de constituer un espace articulé autour d'un projet homogène et cohérent (Djouldem, 1991). C'est cette homogénéisation qui a conduit à l'alignement sur une position radicale. On notera, sur la base de notre étude de cas, que la disposition géographique des principaux porteparole et le rôle des individualités jouent alors un rôle stratégique essentiel. Le réseau est à comprendre ici comme forme d'inscription particulière sur un territoire, comme un quadrillage dans un but de contrôle, voire de pouvoir. Mais réseau et territoire forment un couple conceptuel où les deux termes sont étroitement liés. La socialisation par le réseau, par essence linéaire et lacunaire, permet de dépasser le cadre restreint du territoire (Rodary, 2003, d'après Lévy, 1999). Cela permet de comprendre qu'après avoir tenté une mobilisation à l'échelle de la vallée, du canton ou du département, les faiblesses locales des groupes anti-ours les ont amenés à se structurer de manière linéaire Est-Ouest pour se renforcer. Cette rupture de cadres territoriaux classiques, déclenchée à la base par les déplacements latitudinaux d'ours, s'est peu à peu déployée en une dynamique qui a sa propre logique géopolitique.

Enfin, les actions d'opposition à la conservation de l'ours relèvent-elles d'un mouvement social « spontané » ou « guidé »? Bien sûr, ces deux dimensions sont en général présentes, puisque « les mouvements sociaux sont susceptibles de prendre appui sur des groupes déjà organisés » (Mann, 1990). La question se pose de savoir « quel est alors le critère qui nous permettra de distinguer un mouvement social d'un simple groupe de pression ? » (Ibid). Nous ne proposerons pas ici d'y répondre dans le cas de l'ours. Mais, sur la base de cet exemple, nous pensons avoir montré au moins que les mouvements d'opposition aux actions de conservation, quelle que soit leur part de mobilisation sociale spontanée, ne peuvent pas être compris sans une analyse des stratégies spécifiques, fortement territorialisées, que déploient des acteurs politiques et syndicaux pour intensifier, organiser et utiliser le potentiel de mobilisation hostile que génère, à des degrés divers, toute action de conservation de la nature.

Ce constat et ceux qui ont ponctué les différentes étapes de notre analyse de cas nous conduisent vers des perspectives plus générales.

Dans l'analyse des problèmes de conservation, ils confirment et illustrent la nécessité, voire l'urgence, d'une critique serrée de l'utilisation de la 


\section{Liste des sigles utilisés}

ADET : Association pour le développement économique et touristique en pyrénées centrales

ADIP: Association de défense de l'identité pyrénéenne

ADDIP: Association pour le développement durable de l'identité pyrénéenne

AET 3V : Association des éleveurs transhumants des trois vallées : Ossau, Aspe, Barétous

ASPP : Association pour la Sauvegarde du patrimoine pyrénéen

DDAF : Direction départementale de l'agriculture et des forêts

DIREN : Direction régionale de l'environnement

FDSEA : Fédération départementale des syndicats d'exploitants agricoles

IDAMP : Association interdépartementale de défense de l'agriculture de montagne

IPHB : Institution patrimoniale du Haut Béarn

notion de local et des thématiques qui lui sont habituellement associées. Loin de nous l'idée de contester l'importance de dynamiques locales dans ces questions. Nous voulons seulement souligner le caractère mystificateur des oppositions si souvent martelées entre action de conservation (supposées non locales) et réticences (affichées comme locales), ainsi que l'indigence de certaines notions très floues régulièrement mobilisées dans ce cadre. Nous pensons avoir montré qu'une appréhension utile de ces questions passe d'abord par une critique du local, puis par une reconstruction (encore essentiellement à venir dans le cadre des recherches sur la biodiversité) de conceptions bien choisies et fondées des dimensions territoriales des problèmes d'environnement et de ressources. Dans un premier temps, une telle reconstruction repose sur l'analyse d'emboîtements de plusieurs échelles territoriales et de plusieurs niveaux organisationnels. Mais, dans un second temps, ces emboîtements sont accompagnés d'une mise en évidence des effets et des stratégies en réseau qui enrichissent et/ou dépassent ces relations d'inclusions. Un tel travail est essentiel pour éclairer les processus de décentralisation en cours et à venir dans la gestion des problèmes d'environnement.

L'analyse des oppositions aux actions en faveur de l'ours, que l'on peut extrapoler à la faune, la flore, les milieux, recouvre au moins quatre dimensions :

1. les résistances qui accompagnent nécessairement (au moins dans le principe) toute action portée par un acteur et ayant pour finalité des changements de perception ou de comportement de la part d'autres acteurs ;

2. des oppositions motivées par des divergences frontales d'intérêt (ici, par exemple, de la part d'éleveurs qui souhaitent conserver ou développer certains systèmes d'élevage incompatibles techniquement ou économiquement avec la présence des prédateurs) ;

3. l'utilisation à des fins politiques (ou syndicales) des difficultés et des controverses liées à la gestion de la faune et des milieux (ici, par exemple, l'utilisation du dossier de l'ours pour porter des stratégies de maîtrise politique ou de fédération syndicale agricole à l'échelle du Béarn dans les années 1980, puis de l'ensemble du massif pyrénéen dans les années 90 ) ;

4. les dynamiques qui peuvent amplifier les trois précédentes, en leur surajoutant des conflits interpersonnels, des difficultés de communication interculturelle, certains choix dans l'action de la part des acteurs porteurs du changement, les conséquences d'événements ou de facteurs conjoncturels, etc. (ici, par exemple, l'arrêt des promesses de fonds publics pour une gestion de type IPHB à l'échelle de l'ensemble du massif, ou bien l'errance de l'ours Néré).

Ces dimensions peuvent se combiner en des configurations très diverses, parfois complexes. En revanche, nous voulons souligner qu'elles sont irréductibles l'une à l'autre. Il faut donc considérer avec une vigilance critique particulière les interprétations qui, dans un cas donné, attribueraient les oppositions directement - sans étude empirique adéquate et sans autre forme d'analyse - aux seules erreurs commises par les promoteurs des actions de conservation, et/ou aux seules contraintes économiques ou techniques que ces actions entraîneraient.

Au-delà des discours qui les accompagnent, et qui souvent donnent lieu à de telles confusions, ces oppositions doivent être replacées dans la dynamique d'ensemble où elles trouvent leur sens. Elles doivent être situées dans le contexte stratégique où peuvent se lire les motivations des personnes qui les portent. Nous espérons avoir montré ici que, pour cela, une approche centrée sur les interactions stratégiques entre acteurs et sur la géopolitique des territoires concernés est particulièrement appropriée. La collaboration entre chercheur en gestion et géographe trouve ici tout son intérêt et ne demande qu'à être développée plus avant. 


\section{Références}

ADDIP-IDAMP, 2001. La colère des Pyrénées, Recueil de témoignages.

ASCA, Servheen, C., 1996. État des lieux de la population ursine et de son habitat dans le Haut Béarn. Stratégies de conservation et de renforcement éventuel, Rapport de phase 1, ASCA, commande de l'Institut patrimonial du Haut Béarn, OloronSainte-Marie.

Benhammou, F., 2001. Rôles et dynamiques des porte-parole dans les débats sur l'ours dans les Pyrénées, analyse stratégique et géopolitique d'un problème d'environnement, Mémoire de DEA ADEN. Université d'Orléans, ENGREF, Paris.

Benhammou, F., 2003. Des Hommes, des Ours, des Montagnes. Pour, septembre 2003.

Bourdin, A., 2000. La question locale. PUF, Paris.

Camarra, J.-J., Parde, J. M., 1992. L'Ours (Ursus arctos), Encyclopédie des carnivores de France, 5. SFEPM, Paris.

Caussimont, G., 1993. La population d'Ours brun dans les Pyrénées françaises et espagnoles, historique d'un déclin : l'exemple des Pyrénées occidentales. Acte du XVI colloque de la SFEPM. Grenoble 17-18 octobre 1992, Muséum d'histoire naturelle, Grenoble, pp. 101-106.

Couteau-Bégarie, A., 2001. Traité de stratégie. Economica, Paris.

De Marliave, O., 2000. Histoire de l'ours dans les Pyrénées. Editions du Sud-Ouest, Coll. « Histoire», Toulouse.

Dendaletche, C., 1993. La Cause de l'ours. Sang de la Terre, Paris.

DIREN Midi-Pyrénées, 2000. Bilan public « Ours »- 24 juin 2000. DIREN M-P, Toulouse, Retranscription littérale.
DIREN Midi-Pyrénées, 2002. Programme de restauration de l'ours dans les Pyrénées. Note d'information, 18 octobre 2002.

Djouldem, M., 1991. Le local en réseau. Quaderni 13-14, 25-38.

Lacoste, Y., 1995. Dictionnaire de géopolitique. Flammarion, Paris.

Lacoste, Y., 1997. Vive la nation. Destin d'une idée géopolitique. Fayard, Paris.

Levy, J., 1999. Le tournant géographique. Coll. « Mappemonde ", Belin, Paris.

Mann, P., 1990. Mouvements sociaux. In: Auroux, S. (Ed.), Les notions philosophiques-dictionnaire. PUF, Paris, 1699-1700.

Mermet, L., 1992. Stratégies pour la gestion de l'environnement : la nature comme jeu de société ? L'Harmattan, Paris.

Mermet, L., 1998. L'analyse stratégique de la gestion environnementale - illustrée par les tribulations d'un noyau relictuel de population d'ours brun dans les Pyrénées occidentales françaises. ENGREF-RGTE, Paris.

Mermet, L., 2001. L'Institut Patrimonial du Haut-Béarn : gestion intégrée de l'environnement ou réaction anti-environnementale ? Responsabilité Environnement, janvier 2001, 9-21.

Mermet, L., 2002. L'Homme ou la vie sauvage ? La société locale ou la bureaucratie centrale? Faux dilemmes et vrais rapports de force. Responsabilité Environnement, 13-21 octobre 2002.

Rodary, E., 2003. Pour une géographie politique de l'environnement. Écologie politique 27, 91-111.

Rossi, G., 2001. L'ingérence écologique. CNRS Éditions, Paris.

Rowell, A., 1996. Green backlash - global subversion of the environmental movement. Routledge, Londres.

Soubeyran, O., Barnier, V., 1996. Le cas du tunnel du Somport. Ecodécision, Automne 1996, 66-71.

Available online at www.sciencedirect.com

science@Directo 